\title{
Article \\ Antenatal Betamethasone Every 12 Hours in Imminent Preterm Labour
}

\author{
Natalia Saldaña-García 1,2,* , María Gracia Espinosa-Fernández ${ }^{1}$, Jose David Martínez-Pajares ${ }^{1}$ (D), \\ Elías Tapia-Moreno ${ }^{1}$, María Moreno-Samos ${ }^{1}$, Celia Cuenca-Marín ${ }^{3}$, Francisca Rius-Díaz ${ }^{4}$ (D) \\ and Tomás Sánchez-Tamayo ${ }^{1,5, *}$
}

check for

updates

Citation: Saldaña-García, N.;

Espinosa-Fernández, M.G.;

Martínez-Pajares, J.D.; Tapia-

Moreno, E.; Moreno-Samos, M.;

Cuenca-Marín, C.; Rius-Díaz, F.;

Sánchez-Tamayo, T. Antenatal

Betamethasone Every 12 Hours in

Imminent Preterm Labour. J. Clin.

Med. 2022, 11, 1227. https://doi.org/

$10.3390 / j \mathrm{~cm} 11051227$

Academic Editor: Sylvie Girard

Received: 15 January 2022

Accepted: 22 February 2022

Published: 24 February 2022

Publisher's Note: MDPI stays neutral with regard to jurisdictional claims in published maps and institutional affiliations.

Copyright: (C) 2022 by the authors. Licensee MDPI, Basel, Switzerland. This article is an open access article distributed under the terms and conditions of the Creative Commons Attribution (CC BY) license (https:// creativecommons.org/licenses/by/ $4.0 /)$.
1 Department of Neonatology, Regional University Hospital of Málaga, 29010 Malaga, Spain; mgespinosaf@gmail.com (M.G.E.-F.); jd_martinezp@hotmail.com (J.D.M.-P.); eliastamo@hotmail.com (E.T.-M.); maria.m.samos@gmail.com (M.M.-S.)

2 School of Medicine, Malaga University, 29071 Malaga, Spain

3 Department of Obstetrics and Gineocology, Regional University Hospital of Málaga, 29010 Malaga, Spain; dra.cuencamarin@gmail.com

4 Department of Preventive Medicine and Public Health, Biostatistics, School of Medicine, Malaga University, 29071 Malaga, Spain; rius@uma.es

5 Pharmacology and Pediatrics Department, Malaga University, 29071 Malaga, Spain

* Correspondence: natalia@saldanagarcia.es (N.S.-G.); tomas.sanchez.tamayo@gmail.com (T.S.-T.)

\begin{abstract}
Background: Benefits of antenatal corticosteroids have been established for preterm infants who have received the full course. In imminent preterm labours there is no time to administer the second dose $24 \mathrm{~h}$ later. Objective: To determine whether the administration of two doses of betamethasone in a $12 \mathrm{~h}$ interval is equivalent to the effects of a full maturation. Methods: We performed a retrospective cohort study including preterm infants $\leq 34$ weeks gestational age at birth and $\leq 1500 \mathrm{~g}$, admitted to an NICU IIIC level in a tertiary hospital from 2015 to 2020 . The population was divided into two cohorts: complete maturation (CM) (two doses of betamethasone $24 \mathrm{~h}$ apart), or advanced maturation (AM) (two doses of betamethasone $12 \mathrm{~h}$ apart). The primary outcomes were mortality or survival with severe morbidities. The presence of respiratory distress syndrome and other morbidities of prematurity were determined. These variables were analysed in the neonates under 28 weeks gestational age cohort. Neurodevelopment at 2 years was evaluated with the validated Ages and Stages Questionnaires ${ }^{\circledR}$, Third Edition (ASQ ${ }^{\circledR}-3$ ). Multiple regression analyses were performed and adjusted for confounding factors. Results: A total of 275 preterm neonates were included. Serious outcomes did not show differences between cohorts, no increased incidence of morbidity was found in AM. A lower percentage of hypotension during the first week $(p=0.04)$, a tendency towards lower maximum $\mathrm{FiO}_{2}(p=0.14)$ and to a shorter mechanical ventilation time $(p=0.14)$ were observed for the AM cohort. Similar results were found in the subgroup of neonates under 28 weeks gestational age. There were no differences in cerebral palsy or sensory deficits at 24 months of corrected age, although the AM cohort showed a trend towards better scores on the ASQ3 scale. Conclusions: Administration of betamethasone every $12 \mathrm{~h}$ showed similar results to the traditional pattern with respect to mortality and severe morbidities. No deleterious neurodevelopmental effects were found at 24 months of corrected age. Earlier administration of betamethasone at $12 \mathrm{~h}$ after the first dose would be an alternative in imminent preterm delivery. Further studies are needed to confirm these results.
\end{abstract}

Keywords: antenatal corticosteroids; betamethasone; preterm infant; mortality; respiratory distress syndrome; neurodevelopmental

\section{Introduction}

The discovery of the effects of antenatal corticosteroid administration as a measure for assisting lung maturation in threatened preterm labour, is one of the milestones of modern 
medicine [1]. Since the first investigations in 1970 and 1972 by Liggins and Howie [2], several studies and reviews have been published supporting the use of antenatal corticosteroids $[3,4]$. They have been shown to reduce neonatal and perinatal serious outcomes (defined as death or survival with serious sequelae), neonatal respiratory distress syndrome (RDS) and they probably reduce the occurrence of severe intraventricular haemorrhage (IVH) $[1,4]$.

Guidelines and types of corticosteroids administered have not changed much since their discovery [2]. Current international recommendations are to initiate treatment with betamethasone (12 mg every 24 h, 2 doses) or dexamethasone (6 mg every 12 h, 4 doses) [5], in any gestation at risk of preterm delivery from $24+0$ weeks gestational age (GA) to $34+0$ weeks GA [6,7]. Its administration should be agreed with the parents at gestational ages at the limit of viability $(23+0$ to $23+6)$. In the later preterm population, above $34+0$ weeks GA, its use should be on an individualised basis, and there is currently much controversy in its administration, given that although prenatal corticosteroids reduce respiratory distress syndrome, more studies are needed to prove the effects on neurodevelopment in the longer term [4,7-9].

The benefits of prenatal corticosteroids on preterm lung maturation have been established for those pregnancies who received the full corticosteroid course and ended within $48 \mathrm{~h}$ to 7 days of the first dose of prenatal corticosteroid [1,4]. However, in routine clinical practice, this condition is not always met. More than $50 \%$ of pregnant women who received prenatal corticosteroids continue their pregnancy beyond day 7 from maturity or go to term [10]. It is also estimated that $15 \%$ of pregnant women who received prenatal corticosteroids finish their pregnancy before $48 \mathrm{~h}$ from the start of regimen, sometimes without having received the two doses of betamethasone or four doses of dexamethasone, which complete the course [11]. In these cases of imminent delivery, international guidelines recommend an administration of at least one dose of prenatal corticosteroid. [1,4,12].

Recent studies call for further research on dosing and timing of prenatal corticosteroids to individualise treatment $[4,11,13]$.

In our centre, a tertiary hospital with a Neonatal Intensive Care Unit (NICU) level IIIC [14], maturation with prenatal corticosteroids is performed with betamethasone (50\% in the acetate form and $50 \%$ in the phosphate form). In situations of imminent preterm labour, when it is not possible to wait $24 \mathrm{~h}$ for the administration of the second dose, it is advanced to $12 \mathrm{~h}$ after the first dose to complete the cycle.

The main objective of this study was to determine neonatal serious outcomes (mortality and serious sequelae) in preterm infants who received two doses of betamethasone $12 \mathrm{~h}$ apart compared to preterm infants who received the full standard regimen.

\section{Materials and Methods}

A retrospective cohort study was conducted from 1 January 2015 to 31 December 2020, including preterm infants $\leq 34$ weeks, weighing $\leq 1500 \mathrm{~g}$ admitted to a tertiary hospital at a NICU IIIC level. Neonates with major malformations were excluded from the study.

Two cohorts were established with regard to the type of prenatal corticosteroid maturation received. A complete maturation cohort (CM) included neonates who received 2 doses of antenatal betamethasone $24 \mathrm{~h}$ apart and whose birth occurred between $48 \mathrm{~h}$ and 7 days after the initial dose. The advanced maturation (AM) cohort included preterm infants who received 2 doses of antenatal betamethasone $12 \mathrm{~h}$ apart and whose birth occurred after completion of the regimen and up to 7 days after the initial dose.

The criteria for the betamethasone every $12 \mathrm{~h}$ administration were: situations of imminent labour (cervical length $<15 \mathrm{~mm}$ and regular clinical uterine dynamics despite tocolytic treatment; cervical conditions of delivery and regular clinical uterine dynamics despite tocolytic treatment; extremely advanced labour conditions where, even without uterine dynamics, labour may occur unpredictably); situations of chorioamnionitis or intrauterine growth retardation >stage III, according to Figueras classification [15] and/or with cardiotocography (CTG) recordings that are not very reassuring and situations of 
maternal pathology in which labour is decided to be terminated within $24 \mathrm{~h}$ of the first dose of betamethasone.

The following variables were recorded as demographic and clinical characteristics: maternal age, primiparity, single/multiple gestation, the presence of gestational diabetes, hypertensive stages including pre-eclampsia, eclampsia and HELLP syndrome, intrauterine growth restriction (IUGR), chorioamnionitis, and third trimester haemorrhage (placental abruption, placenta praevia, uterine rupture or vasa praevia). The following data were collected: caesarean section, gestational age (GA) in weeks, anthropometric measurements at birth, gender. The difference in hours between the first dose of corticosteroids and delivery was also determined.

In our centre, low-dose postnatal hydrocortisone was administered during the first 10 days of life in neonates under 26 weeks and between $26+0$ and $27+6$ weeks if they presented with chorioamnionitis or had not received prenatal corticosteroids, according to the PREMILOC protocol. The study by Baud et al. (2016) aims to increase survival and improve outcomes in bronchopulmonary dysplasia in neonates under 28 weeks gestational age [16]. Patients who received this guideline in each cohort are included in our study.

The main objective was to determine "serious outcomes", defined as death or surviving neonates with serious sequelae, which included the following: intraventricular haemorrhage grade III-IV (IVH), periventricular leukomalacia (PVL), bronchopulmonary dysplasia (BPD), necrotising enterocolitis (NEC) stage $>2$, retinopathy of prematurity (ROP) requiring treatment. These variables were defined with regard to the criteria established by the Vermont Oxford working group $[17,18]$.

As secondary outcomes, intubation required at birth was determined, Apgar score $<5$ at 5 min of life, the need for mechanical ventilation (MV), maximum $\mathrm{FiO}_{2}$ during admission and need for surfactant administration. Other variables analysed included hypotension during the first week of life requiring treatment (volume expansion or inotropic administration), patent ductus arteriosus requiring treatment, spontaneous bowel perforation and the presence of early sepsis or late sepsis $[17,18]$.

A sub-analysis of the variables was performed for neonates under 28 weeks gestational age.

Finally, both cohorts were monitored up to 24 months corrected age. The validated Ages and Stages Questionnaires ${ }^{\circledR}$, Third Edition (ASQ ${ }^{\circledR}-3$ ) were used to assess neurodevelopmental status $[19,20]$. The presence of cerebral palsy was determined, defined as chronic, non-progressive impairment of motor skills, posture, balance, coordination, tone or reflexes. The presence of sensory deficits was analysed: visual (need for correcting lenses or mono/bilateral blindness) and auditory (need for hearing aids or mono/bilateral deafness).

Contingency tables and the chi-squared test were used for the comparison of qualitative variables. In the $2 \times 2$ tables with a low number of observations $(n<5)$, Fisher's exact test was used. To carry out pairwise comparisons of quantitative variables, the Student's $t$-test or the Mann-Whitney U test was used, depending on the distribution of the variables. For variables showing an association with $p<0.25$, a multivariate model was adjusted for the following confounding factors: GA, IUGR, third trimester haemorrhage, chorioamnionitis, hypertensive stages, Apgar test score $<5$ at 5 min of life, gender and postnatal hydrocortisone treatment. The backward method selection was used, variables with $p$ values $\geq 0.15$ for the Wald statistic were removed from the model until the adjusted OR estimate was obtained. In all cases, a statistically significant difference was declared when $p<0.05$. SPSS v25.0 (IBM Corp., Armonk, NY, USA) was used for this analysis.

This study was approved by the Provincial Ethics Committee of Málaga and by the Medical Management of the Regional University Hospital of Málaga, dated 12 November 2020. 


\section{Results}

A total of 275 patients were studied, and were divided into the complete maturation $(\mathrm{CM})$ cohort $(n=224)$ and the 2 doses $12 \mathrm{~h}$ apart $(\mathrm{AM})$ cohort $(n=51)$. The clinical and demographic characteristics of the two cohorts are summarised in Table 1.

Table 1. Cohort characteristics with regard to the interval between antenatal corticosteroid doses.

\begin{tabular}{|c|c|c|c|}
\hline & $\begin{array}{c}\text { CM } \\
N=224\end{array}$ & $\begin{array}{c}\mathrm{AM} \\
N=\mathbf{5 1}\end{array}$ & $\begin{array}{c}\text { Significance } \\
(p)^{*}\end{array}$ \\
\hline Maternal age (years) & $32.86 \pm 6.22$ & $31.80 \pm 5.46$ & 0.23 \\
\hline Multiple gestation & $49(21.9 \%)$ & $17(33.3 \%)$ & 0.08 \\
\hline Gestational diabetes & $6(3.7 \%)$ & $4(9.3 \%)$ & 0.11 \\
\hline Hypertensive states & $92(41.4 \%)$ & $12(23.5 \%)$ & 0.03 \\
\hline Chorioamnionitis & $45(20.1 \%)$ & $15(29.4 \%)$ & 0.14 \\
\hline Premature rupture of membranes & $68(30.4 \%)$ & $16(31.4 \%)$ & 0.88 \\
\hline Third trimester haemorrhage & $27(12 \%)$ & $3(5.9 \%)$ & 0.21 \\
\hline IUGR & $77(34.4 \%)$ & $10(19.6 \%)$ & 0.04 \\
\hline Caesarean section & $184(82.2 \%)$ & $39(76.5 \%)$ & 0.75 \\
\hline Gestational age at first dose & $27.9 \pm 0.19$ & $28.5 \pm 0.38$ & 0.14 \\
\hline Difference in hours from the first dose to birth & $89.5(59-122.7)$ & $20(16-30)$ & $<0.001$ \\
\hline Difference in hours from the last dose to birth & $65(35-98.7)$ & $4(2-12)$ & $<0.001$ \\
\hline Gestational age (weeks) & $28.5 \pm 0.2$ & $28.7 \pm 0.3$ & 0.53 \\
\hline Weight at birth (grams) & $991.4 \pm 20.9$ & $1087.2 \pm 42.1$ & 0.04 \\
\hline Length at birth $(\mathrm{cm})$ & $35.5 \pm 0.3$ & $36.8 \pm 0.6$ & 0.05 \\
\hline Head circumference at birth & $25.4 \pm 0.2$ & $25.8 \pm 0.3$ & 0.39 \\
\hline Female gender & $104(46.4 \%)$ & $31(60.8 \%)$ & 0.06 \\
\hline Postnatal hydrocortisone & $22(9.8 \%)$ & $8(15.7 \%)$ & 0.22 \\
\hline
\end{tabular}

Results for continuous variables are expressed as mean \pm standard deviation, and as median and interquartile range. Qualitative variables show total number and percentage relative to their cohort. * Chi-square, Student's $t$-test, Mann-Whitney U. CM: complete maturation; AM: advance maturation. IUGR: Intrauterine growth restriction.

The percentage of intrauterine growth retardation and hypertensive stages were more frequent in the CM group. Gestational age at the first dose of betamethasone and gestational age at birth were similar in both cohorts. The CM cohort presented with lower weight and height at birth, with no differences in head circumference, in relation to the higher percentage of IUGR. The differences in hours from first and second dose to delivery are summarised. Gestational age at first dose of betamethasone is summarised, no differences were found.

There are no significant differences in other population characteristics, postnatal corticosteroid administration included.

The results of the overall analysis of both cohorts are summarised in Table 2. For serious outcomes, no differences were found between the two cohorts, either the components analysed individually (mortality, PVL, IVH III-IV grade, treated ROP, NEC > 2nd grade and moderate-severe BPD).

With regard to the assessment of respiratory distress, no differences were found in the need for mechanical ventilation during admission, nor in the surfactant administration. Lower peak $\mathrm{FiO}_{2}$ and shorter mechanical ventilation time were observed in the advanced maturation (AM) cohort. When it was adjusted for confounding factors, significance was lost.

A notable finding was the lower percentage of hypotension during the first week in the AM cohort. When it was adjusted for confounding factors OR 0.5 (95\%CI 0.3-0.9), it was maintained.

No cases of spontaneous intestinal perforations were reported in the AM cohort, compared to 13 cases (5.8\%) in the CM cohort. There were no significant differences in cases with presence of early or late sepsis.

The variables analysed for the group under 28 weeks GA showed similar results in terms of serious outcomes and severe morbidities of prematurity (Table 3 ). Lower 
maximum $\mathrm{FiO}_{2}$ was found for the $\mathrm{AM}$ cohort. Lower hypotension during the first week of life and shorter mechanical ventilation time were found in favour of the AM group. This significance was lost in the adjusted model; however, the model decreased its goodness of fit by $12 \%$ for hypotension and $50 \%$ for mechanical ventilation time when prenatal corticosteroid therapy received, was removed from the equation.

Table 2. Results of the cohort comparison by prenatal corticosteroid inter-dose interval.

\begin{tabular}{|c|c|c|c|c|c|c|}
\hline & $\begin{array}{c}\text { CM } \\
N=224\end{array}$ & $\begin{array}{c}\text { AM } \\
N=51\end{array}$ & $\begin{array}{l}\text { OR (CI 95\%) } \\
\text { Unadjusted }\end{array}$ & $p^{*}$ & $\begin{array}{c}\text { OR (CI 95\%) } \\
\text { Adjusted }\end{array}$ & Adjusted $p^{\dagger}$ \\
\hline Serious outcome & $89(39.7 \%)$ & $17(33.3 \%)$ & $0.7(0.4-1.4)$ & 0.39 & & \\
\hline Neonatal death & $31(13.8 \%)$ & $4(7.8 \%)$ & $0.5(0.17-1.57)$ & 0.24 & $0.5(0.1-1.8)$ & 0.36 \\
\hline Intubation at birth & $125(55.8 \%)$ & $27(52.9 \%)$ & $0.9(0.4-1.6)$ & 0.71 & & \\
\hline Apgar $<5$ at $5 \mathrm{~min}$ & $16(7.1 \%)$ & $2(3.9 \%)$ & $0.5(0.1-2.3)$ & 0.40 & & \\
\hline Severe IVH & $19(8.5 \%)$ & $4(7.8 \%)$ & $0.9(0.3-2.8)$ & 0.88 & & \\
\hline PVL & $8(3.6 \%)$ & $1(2 \%)$ & $0.5(0.06-4.4)$ & 0.56 & & \\
\hline $\mathrm{NEC}>2$ & $5(2.2 \%)$ & 0 & $0.8(0.7-0.8)$ & 0.28 & & \\
\hline Intestinal perforation & $13(5.8 \%)$ & 0 & $0.8(0.7-0.8)$ & 0.13 & NA & NA \\
\hline Treated ROP & $30(13.4 \%)$ & $6(11.8 \%)$ & $0.8(0.3-2.1)$ & 0.75 & & \\
\hline Hypotension 1 week & $60(26.8 \%)$ & $5(10 \%)$ & $0.3(0.1-0.8)$ & 0.01 & $0.5(0.3-0.9)$ & 0.04 \\
\hline Treated PDA & $20(8.9 \%)$ & $7(13.7 \%)$ & $1.6(0.6-4.1)$ & 0.29 & & \\
\hline Early sepsis & $13(5.8 \%)$ & $6(12 \%)$ & $2.2(0.7-6.1)$ & 0.11 & $2.3(0.7-7.2)$ & 0.13 \\
\hline Late sepsis & $99(44.2 \%)$ & $17(34 \%)$ & $0.6(0.3-1.2)$ & 0.18 & $0.8(0.4-1.7)$ & 0.63 \\
\hline BPD & $50(25.1 \%)$ & $8(17 \%)$ & $0.6(0.2-1.3)$ & 0.23 & $0.6(0.2-1.8)$ & 0.44 \\
\hline Need for surfactant & $122(54.5 \%)$ & $28(54.9 \%)$ & $1.01(0.5-1.8)$ & 0.95 & & \\
\hline Need for MV & $103(46 \%)$ & $21(41.2 \%)$ & $0.8(0.4-1.5)$ & 0.53 & & \\
\hline Maximum $\mathrm{FiO}_{2}$ & $42.6 \pm 2.1$ & $34.8 \pm 3.4$ & & 0.05 & & 0.14 \\
\hline MV time (hours) & $361.7 \pm 58.5$ & $191.1 \pm 47.1$ & & 0.02 & & 0.14 \\
\hline
\end{tabular}

Qualitative variables are expressed as $\mathrm{n}(\%)$ within each cohort, quantitative variables as mean \pm standard deviation. * Chi-square, Fisher's exact test, Student's $t$-test. + Results of multivariate analyses are shown for significant variables in the unadjusted model.CM: complete maturation; AM: advanced maturation. IVH: intraventricular haemorrhage; PVL: periventricular leukomalacia; NEC: necrotising enterocolitis; ROP: retinopathy of prematurity; PDA: patent ductus arteriosus; BPD: bronchopulmonary dysplasia; MV: mechanical ventilation. NA: not applicable.

Table 3. Outcomes in $<28$ weeks gestational age according to interval between doses of antenatal corticosteroids.

\begin{tabular}{|c|c|c|c|c|c|c|}
\hline & $\begin{array}{c}\text { CM } \\
N=94\end{array}$ & $\begin{array}{c}\mathrm{AM} \\
N=19\end{array}$ & $\begin{array}{l}\text { OR (CI 95\%) } \\
\text { Unadjusted }\end{array}$ & $p^{*}$ & $\begin{array}{c}\text { OR (CI 95\%) } \\
\text { Adjusted }\end{array}$ & $\begin{array}{c}p^{\dagger} \\
\text { Adjusted }\end{array}$ \\
\hline Serious outcome & $70(74.5 \%)$ & $13(68.4 \%)$ & $0.7(0.2-2.1)$ & 0.58 & & \\
\hline Neonatal death & $26(27.7 \%)$ & $3(15.8 \%)$ & $0.4(0.1-1.8)$ & 0.32 & & \\
\hline Intubation at birth & $86(91.5 \%)$ & $18(94.7 \%)$ & $1.6(0.1-14.2)$ & 0.99 & & \\
\hline Apgar $<5$ at $5 \mathrm{~min}$ & $11(11.7 \%)$ & $1(5.3 \%)$ & $0.4(0.05-3.4)$ & 0.41 & & \\
\hline Severe IVH & $16(17 \%)$ & $3(15.8 \%)$ & $0.9(0.2-3.5)$ & 0.89 & & \\
\hline PVL & $7(7.4 \%)$ & 0 & $0.6(0.3-1.3)$ & 0.59 & & \\
\hline $\mathrm{NEC}>2$ & - & - & - & - & & \\
\hline Intestinal perforation & $11(11.7 \%)$ & 0 & $0.4(0.2-0.8)$ & 0.01 & NA & \\
\hline Treated ROP & $27(28.7 \%)$ & $4(21.1 \%)$ & $0.6(0.2-2.1)$ & 0.49 & & \\
\hline Hypotension 1 week & $49(52.1 \%)$ & $3(16.7 \%)$ & $0.1(0.05-0.6)$ & 0.009 & $0.2(0.05-1)$ & 0.06 \\
\hline Treated PDA & $18(19.1 \%)$ & $6(31.6 \%)$ & $1.9(0.6-5.8)$ & 0.22 & & \\
\hline Early sepsis & $11(11.7 \%)$ & $2(11.1 \%)$ & $0.9(0.1-4.6)$ & 0.99 & & \\
\hline Late sepsis & $61(64.9 \%)$ & $11(61.1 \%)$ & $0.8(0.3-2.4)$ & 0.75 & & \\
\hline BPD & $41(56.2 \%)$ & $7(43.8 \%)$ & $0.6(0.2-1.8)$ & 0.36 & & \\
\hline Need for surfactant & $85(90.4 \%)$ & $17(89.5 \%)$ & $0.9(0.1-4.5)$ & 0.89 & & \\
\hline Need for MV & $68(72.3 \%)$ & $12(63.2 \%)$ & $0.6(0.2-1.8)$ & 0.42 & & \\
\hline Maximum $\mathrm{FiO}_{2}$ & $62.2 \pm 3.4$ & $44.4 \pm 7.2$ & & 0.03 & & 0.04 \\
\hline MV time (hours) & $477.8 \pm 82.4$ & $203.1 \pm 51.6$ & & 0.006 & & 0.14 \\
\hline
\end{tabular}

Qualitative variables are expressed as $\mathrm{n}(\%)$ within each cohort, quantitative variables such as mean \pm standard deviation. * Chi-square, Fisher's exact test, Student's $t$-test. ${ }^{+}$Results of multivariate analyses are shown for significant variables in the unadjusted model. vIVH: intraventricular haemorrhage; PVL: periventricular leukomalacia; NEC: necrotising enterocolitis; ROP: retinopathy of prematurity; PDA: patent ductus arteriosus; BPD: bronchopulmonary dysplasia; MV: mechanical ventilation. NA: not applicable. CM: complete maturation; AM: advanced maturation. 
The results of the assessment at 24 months corrected age are summarised in Table 4 . No significant differences were found between the cohorts in terms of presence of cerebral palsy, visual or hearing impairment.

Table 4. 24 months corrected age monitoring results, according to antenatal corticosteroid regimen.

\begin{tabular}{|c|c|c|c|c|c|c|}
\hline & $\begin{array}{c}\text { CM } \\
N=101\end{array}$ & $\begin{array}{c}\text { AM } \\
N=22\end{array}$ & OR (CI 95\%) & $p^{*}$ & $\begin{array}{c}\text { OR (CI 95\%) } \\
\text { Adjusted }\end{array}$ & $\begin{array}{c}p^{\dagger} \\
\text { Adjusted }\end{array}$ \\
\hline Cerebral palsy & $3(3 \%)$ & $1(4.5 \%)$ & $1.5(0.1-15.7)$ & 0.71 & & \\
\hline Hearing impairment & $6(6 \%)$ & $3(14.3 \%)$ & $2.6(0.6-11.4)$ & 0.18 & $3.2(0.7-14.9)$ & 0.13 \\
\hline Visual impairment & $11(10.9 \%)$ & $1(4.5 \%)$ & $2.4(0.3-19.9)$ & 0.68 & & \\
\hline \multicolumn{7}{|l|}{ Score below LIN } \\
\hline ASQ-communication & $19(20.2 \%)$ & $2(10 \%)$ & $0.4(0.9-2.1)$ & 0.35 & & \\
\hline ASQ-gross-motor & $10(10.6 \% 9$ & $1(5 \%)$ & $0.4(0.05-3.6)$ & 0.68 & & \\
\hline ASQ-fine-motor & $8(8.5 \%)$ & - & $0.5(0.2-1.1)$ & 0.34 & & \\
\hline ASQ-solving & $9(9.7 \%)$ & $1(5 \%)$ & $0.4(0.05-4.11)$ & 0.68 & & \\
\hline ASQ-social & $12(12.8)$ & $1(5 \%)$ & $0.3(0.04-2.9)$ & 0.45 & & \\
\hline \multicolumn{7}{|l|}{ Average score } \\
\hline ASQ-communication & $43.6 \pm 1.7$ & $48.7 \pm 3.2$ & & 0.17 & & 0.33 \\
\hline ASQ-gross-motor & $50.1 \pm 1.1$ & $51 \pm 3.04$ & & 0.77 & & 0.95 \\
\hline ASQ-fine-motor & $49.8 \pm 1.04$ & $53.5 \pm 1.2$ & & 0.03 & & 0.21 \\
\hline ASQ-P. solving & $45.05 \pm 1.2$ & $48 \pm 1.8$ & & 0.20 & & 0.45 \\
\hline ASQ-P. social & $46.2 \pm 1.2$ & $52 \pm 1.7$ & & 0.01 & & 0.06 \\
\hline
\end{tabular}

Qualitative variables are expressed as n (\%) within each cohort, quantitative variables as mean \pm standard deviation. * Chi-square, Fisher's exact test, Student's $t$-test. ${ }^{\dagger}$ Results of multivariate analyses are shown for significant variables in the unadjusted model. LIN: lower limit of normality. CM: complete maturation; AM: advanced maturation.

The results of the $\mathrm{ASQ}^{\circledR}-3$ questionnaires were shown in two different ways. On the one hand, neonates who scored below the lower limit of normal on each rating scale (communication, gross-motor, fine-motor, problem-solving and social) were determined with regard to the type of maturation received. No significant differences were found between the two cohorts analysed, although the AM population had fewer patients below the lower limit of normal in all categories. On the other hand, mean scores were determined for each category analysed, with regard to the maturation pattern. In all items, the AM cohort scored better, having achieved significance for fine-motor skills and social skills. Statistical significance was lost when the model was adjusted for confounding factors, although in social skills it was close to significance $(p=0.06)$. Figure 1 , box and whisker plot, shows the distribution of the population analysed with respect to the $A S Q^{\circledR}-3$ items, with regard to the type of maturation pattern. The AM cohort showed better scores in all categories.

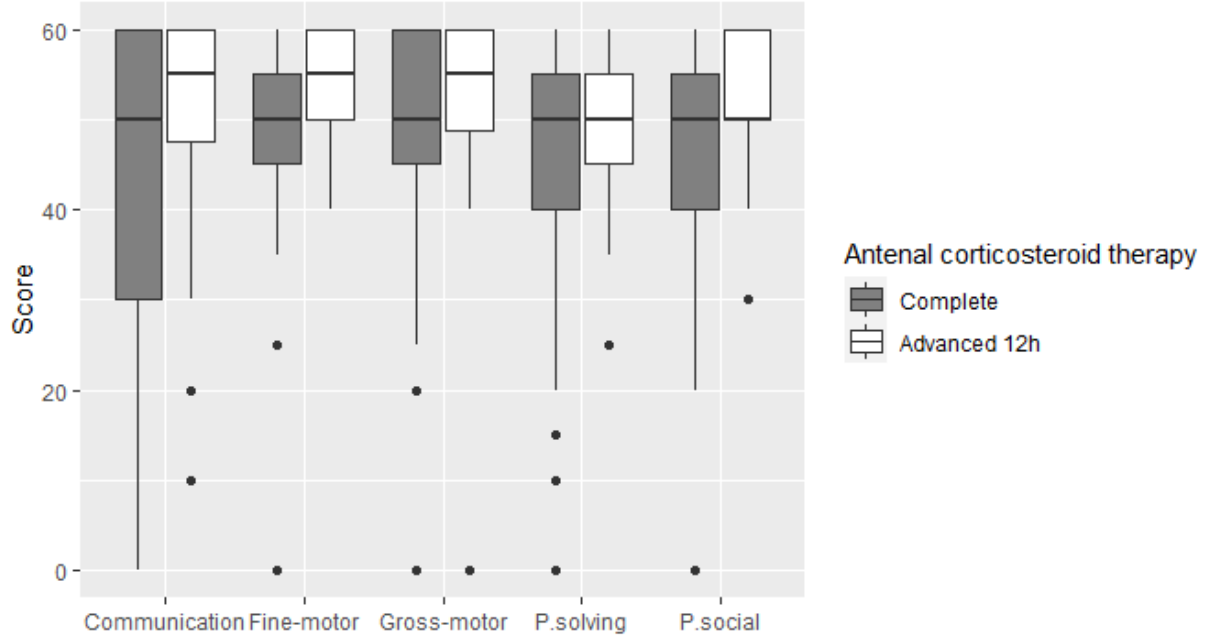

Figure 1. Distribution of the cohorts with respect to the $\mathrm{ASQ}^{\circledR}-3$ scale. P.solving, problem solving; P.social, Personal social. 


\section{Discussion}

The benefits of prenatal corticosteroid administration in case of preterm birth are established for a regimen of betamethasone $12 \mathrm{mg}$ every $24 \mathrm{~h}$ ( 2 doses) or dexamethasone $6 \mathrm{mg}$ every $12 \mathrm{~h}$ (4 doses), with birth occurring from $48 \mathrm{~h}$ after the start of the regimen until the following 7 days [4].

This ideal situation does not always occur in clinical practice where, due to multiple factors, labour may end without having completed the prenatal corticosteroid regimen.

In our study, the effects of a full course of prenatal betamethasone were compared with a $12 \mathrm{~h}$ early course in situations of imminent labour.

Among the baseline characteristics of the population, a higher percentage of hypertensive stages, female gender and intrauterine growth retardation were observed in the AM cohort, so these characteristics were added as confounding factors in the multivariate analysis. With respect to the time elapsed between administration of the first and last dose of betamethasone and delivery, the differences observed are specific to the nature of each cohort.

The results shown were similar in both populations. No harm was found in terms of serious outcomes or respiratory distress syndrome, with respect to complete maturation.

Other studies have analysed the advanced $12 \mathrm{~h}$ schedule of antenatal betamethasone in a randomised clinical trial compared to the usual $24 \mathrm{~h}$ schedule. The study published by Kashanian et al. (2018) included 101 preterm infants in the $12 \mathrm{~h}$ group vs. 100 preterm infants in the $24 \mathrm{~h}$ group. Less respiratory distress and severe intraventricular haemorrhage were found in the $12 \mathrm{~h}$ group, but also higher mortality and necrotising enterocolitis $>2 \mathrm{nd}$ grade. However, there was discordance in gestational ages between groups [21]. In our study, where gestational age was similar between the two cohorts, no differences were found in intraventricular haemorrhage or other morbidities. A better respiratory outcome in the 2 doses $12 \mathrm{~h}$ group was shown in our study, with lower maximum $\mathrm{FiO}_{2}$ during admission and shorter mechanical ventilation time, although both groups had equivalent intubation and surfactant administration requirements. These data were repeated in the subgroup of neonates younger than 28 weeks.

Another clinical trial published by Khandelwald et al. (2012) included 161 preterm infants in the $12 \mathrm{~h}$ betamethasone group vs. 77 preterm infants in the $24 \mathrm{~h}$ betamethasone group [22]. They divided the sample with regard to gestational age subgroups. Mortality, respiratory distress syndrome and other morbidities in preterm infants were analysed. They found similar results in both cohorts, with an increase in the incidence of necrotising enterocolitis $>2$ nd grade. In our study, there were no cases of necrotising enterocolitis or spontaneous intestinal perforation in the $12 \mathrm{~h}$ group.

The haemodynamic effect of corticosteroids at foetal and postnatal level is well known [23-29]. The results of our investigation found greater haemodynamic stability, and a tendency to a better evolution of respiratory distress syndrome in the $12 \mathrm{~h}$ betamethasone group. These results were also verified for the subgroup of neonates under 28 weeks. Studies in lambs have established that the minimum prenatal corticosteroids effective dose to accelerate lung maturation is between $1-4 \mathrm{ng} / \mathrm{mL}$. The current betamethasone regimen reaches concentrations of up to $10 \mathrm{ng} / \mathrm{mL}$ in the first hours of administration, decreasing progressively until $24 \mathrm{~h}$, but maintaining the therapeutic range between $1-4 \mathrm{ng} / \mathrm{mL}$ [13]. The better respiratory and haemodynamic outcomes of preterm infants in the AM cohort may be related to the higher accumulation of prenatal corticosteroid doses. Haemodynamic evolution during the first week of life has not been previously analysed in the clinical studies referred $[5,21,22]$. In our centre, hydrocortisone is administered at low doses during the first ten days of life, to achieve a lower rate of bronchopulmonary dysplasia, according to the PREMILOC protocol [16]. The possible haemodynamic effect of the application of this protocol was controlled in our sample by multivariate analysis, adding it as a confounding factor. The results persisted in favour of the AM group.

The higher percentage of hypertensive stages observed in the CM cohort and therefore of neonates exposed to prenatal antihypertensive treatment (labetalol, methyldopa, 
hydralazine or magnesium sulphate) may influence the higher percentage of neonatal hypotension observed in this cohort [30]. However, after adjusting for confounding factors in the multivariate analysis, including hypertensive stages, significant differences persisted. To confirm our results, a sub-analysis was performed excluding hypertensive stages from the sample, and found a percentage of hypotension of $28.8 \%$ in the CM cohort $(\mathrm{N}=132)$ vs. $7.7 \%$ in the AM cohort $(\mathrm{N}=39)$, with a $p=0.007$.

There are no published studies comparing the evolution of a standard complete maturation pattern with an advanced maturation pattern, with regard to monitoring at 24 months corrected age. Uncertainty about the possible deleterious effect of prenatal corticosteroids on neurodevelopment is a current problem [31,32]. No differences in sensory deficits or increased incidence of infantile cerebral palsy were found in our population. The ASQ3 questionnaire is a validated scale that provides accurate information on development in several areas: communication, gross-motor skills, fine-motor skills, problem solving and personal and social skills [33]. The scores obtained in both cohorts were similar, with a tendency towards better results in the AM group. From a neurological point of view, advancing the dose of betamethasone by $12 \mathrm{~h}$ did not harm our population.

Our study has several limitations to be considered. It is a retrospective cohort study, so we cannot definitively conclude that both betamethasone (every $24 \mathrm{~h}$ or every $12 \mathrm{~h}$ ) treatments are similar. To confirm our results, a clinical trial of equivalence or non-inferiority should be performed.

The baseline population of both cohorts presents some differences: a higher percentage of female sex in the $12 \mathrm{~h}$ cohort, and a higher percentage of intrauterine growth restriction in the complete maturation cohort.

The sample size to detect a $5 \%$ difference in serious outcomes, with a $95 \%$ confidence level, should include at least 808 patients in each cohort., taking as a reference the percentage of serious outcome found in each cohort [34]. Our study included 275 neonates, so the results should be interpreted with caution.

One of the strengths of our study is that, being single-centred, diagnostic and therapeutic criteria are homogenous. Possible confounding factors (gestational age, female gender, percentage of intrauterine growth retardation, reason for prematurity, Apgar score $<5$ at 5 min of life and postnatal hydrocortisone administration) were controlled by multivariate analysis. Both cohorts showed homogeneous results in most of variables analysed and no adverse results were shown in the AM group. This is the only study with monitoring at 24 months corrected age we could find in the literature consulted.

\section{Conclusions}

The administration of antenatal betamethasone $12 \mathrm{mg} 12 \mathrm{~h}$ in advance, has similar serious outcomes to the standard maturation regimen every $24 \mathrm{~h}$ in the preterm infant population analysed. In addition, the advance maturation shows lower hypotension during the first week of life, lower maximum $\mathrm{FiO}_{2}$ during admission and shorter mechanical ventilation time in the analysed population. No deleterious effect on neurodevelopment at 24 months corrected age was found in the AM group. This regimen may be an alternative in situations of imminent preterm labour where there is no time to administer the second dose within $24 \mathrm{~h}$ of the first dose. Nevertheless, randomised clinical trials and long-term neurodevelopmental monitoring are needed to confirm these results.

Author Contributions: Conceptualization, N.S.-G., T.S.-T. and M.G.E.-F.; methodology, J.D.M.-P.; validation, N.S.-G. and F.R.-D.; formal analysis, N.S.-G. and F.R.-D.; resources, C.C.-M.; data curation, N.S.-G., E.T.-M. and M.M.-S.; writing-original draft preparation, N.S.-G.; writing-review and editing, N.S.-G., J.D.M.-P. and M.G.E.-F.; supervision, T.S.-T. All authors have read and agreed to the published version of the manuscript.

Funding: This research received no external funding. 
Institutional Review Board Statement: The study was conducted according to the guidelines of the Declaration of Helsinki and approved by the Provincial Ethics Committee of Málaga and the Medical Management of the Regional University Hospital of Málaga (protocol code TD-CTR; date of approval 11 November 2020).

Informed Consent Statement: Informed consent was obtained from all subjects involved in the study.

Data Availability Statement: The data presented in this study are available on request from the corresponding author. The data are not publicly available due to data protection policies.

Conflicts of Interest: The authors declare no conflict of interest.

\section{References}

1. Briceño-Pérez, C.; Reyna-Villasmil, E.; Vigil De Gracia, P. Antenatal corticosteroid therapy: Historical and scientific basis to improve preterm birth management. Eur. J. Obstet. Gynecol. Reprod. Biol. 2019, 234, 32-37. [CrossRef] [PubMed]

2. Liggins, G.C.; Howie, R.N. A controlled trial of antepartum glucocorticoid treatment for prevention of the respiratory distress syndrome in premature infants. Pediatrics 1972, 50, 515-525. [CrossRef] [PubMed]

3. Crowley, P. Prophylactic corticosteroids for preterm birth. Cochrane Database Syst. Rev. 1996, 1, CD000065. [CrossRef]

4. McGoldrick, E.; Stewart, F.; Parker, R.; Dalziel, S.R. Antenatal corticosteroids for accelerating fetal lung maturation for women at risk of preterm birth. Cochrane Database Syst. Rev. 2020, 12, CD004454. [PubMed]

5. Brownfoot, F.C.; Gagliardi, D.I.; Bain, E.; Middleton, P.; Crowther, C.A. Different corticosteroids and regimens for accelerating fetal lung maturation for women at risk of preterm birth. Cochrane Database Syst. Rev. 2013, 8, CD006764. [CrossRef]

6. El-Sayed, Y.; Borders, A.; Gyamfi-Bannerman, C. Committee on Obstetric Practice. Committee Opinion No. 713: Antenatal Corticosteroid Therapy for Fetal Maturation. Obs. Gynecol. 2017, 130, e102-e109.

7. Obstetricia SEdGy. Guía de Asistencia Práctica Parto pretérmino. Prog. Obstet. Y Ginecol. 2020, 63, $274-346$.

8. Haviv, H.R.; Said, J.; Mol, B.W. The place of antenatal corticosteroids in late preterm and early term births. Semin. Fetal Neonatal Med. 2019, 24, 37-42. [CrossRef]

9. Booker, W.A.; Gyamfi-Bannerman, C. Antenatal Corticosteroids: Who Should We Be Treating? Clin. Perinatol. 2018, 45, 181-198. [CrossRef]

10. Makhija, N.K.; Tronnes, A.A.; Dunlap, B.S.; Schulkin, J.; Lannon, S.M. Antenatal corticosteroid timing: Accuracy after the introduction of a rescue course protocol. Am. J. Obstet. Gynecol. 2016, 214, 120.e1-120.e16. [CrossRef]

11. Hrabalkova, L.; Takahashi, T.; Kemp, M.W.; Stock, S.J. Antenatal Corticosteroids for Fetal Lung Maturity-Too Much of a Good Thing? Curr. Pharm. Des. 2019, 25, 593-600. [CrossRef] [PubMed]

12. Skoll, A.; Boutin, A.; Bujold, E.; Burrows, J.; Crane, J.; Geary, M.; Jain, V.; Lacaze-Masmonteil, T.; Liauw, J.; Mundle, W.; et al. No. 364-Antenatal Corticosteroid Therapy for Improving Neonatal Outcomes. J. Obstet. Gynaecol. Can. 2018, 40, 1219-1239. [CrossRef] [PubMed]

13. Jobe, A.H.; Kemp, M.; Schmidt, A.; Takahashi, T.; Newnham, J.; Milad, M. Antenatal corticosteroids: A reappraisal of the drug formulation and dose. Pediatr. Res. 2021, 89, 318-325. [CrossRef] [PubMed]

14. Lorenzo, F., Jr.; Urcelay, E. Health care levels and minimum recommendations for neonatal care. An. Pediatr. 2013, 79, 51.e1-51.e11.

15. Figueras, F.; Gratacós, E. Update on the diagnosis and classification of fetal growth restriction and proposal of a stage-based management protocol. Fetal Diagn. Ther. 2014, 36, 86-98. [CrossRef] [PubMed]

16. Baud, O.; Maury, L.; Lebail, F.; Ramful, D.; El Moussawi, F.; Nicaise, C.; Zupan-Simunek, V.; Coursol, A.; Beuchée, A.; Bolot, P.; et al. Effect of early low-dose hydrocortisone on survival without bronchopulmonary dysplasia in extremely preterm infants (PREMILOC): A double-blind, placebo-controlled, multicentre, randomised trial. Lancet 2016, 387, 1827-1836. [CrossRef]

17. Horbar, J.D.; Badger, G.J.; Carpenter, J.H.; Fanaroff, A.A.; Kilpatrick, S.; LaCorte, M.; Phibbs, R.; Soll, R.F. Trends in Mortality and Morbidity for Very Low Birth Weight Infants, 1991-1999. Pediatrics 2002, 110, 143-151. [CrossRef]

18. Coll, E.; Jiménez, R. SEN1500: Design and implementation of a registry of infants weighing less than $1500 \mathrm{~g}$ at birth in Spain. An. Pediatr. 2008, 68, 181-188.

19. Steenis, L.J.; Verhoeven, M.; Hessen, D.J.; van Baar, A.L. Parental and professional assessment of early child development: The ASQ-3 and the Bayley-III-NL. Early Hum. Dev. 2015, 91, 217-225. [CrossRef]

20. Sheldrick, R.C.; Marakovitz, S.; Garfinkel, D.; Carter, A.S.; Perrin, E.C. Comparative Accuracy of Developmental Screening Questionnaires. JAMA Pediatr. 2020, 174, 366-374. [CrossRef] [PubMed]

21. Kashanian, M.; Eshraghi, N.; Sheikhansari, N.; Bordbar, A.; Khatami, E. Comparison between two doses of betamethasone administration with 12 hours vs. 24 hours intervals on prevention of respiratory distress syndrome: A randomised trial. J. Obstet. Gynaecol. 2018, 38, 770-776. [CrossRef] [PubMed]

22. Khandelwal, M.; Chang, E.; Hansen, C.; Hunter, K.; Milcarek, B. Betamethasone dosing interval: 12 or 24 hours apart? A randomized, noninferiority open trial. Am. J. Obstet. Gynecol. 2012, 206, 201.e1-201.e11. [CrossRef] [PubMed]

23. Dempsey, E.M. What Should We Do about Low Blood Pressure in Preterm Infants. Neonatology 2017, 111, 402-407. [CrossRef]

24. Agnew, E.J.; Ivy, J.R.; Stock, S.J.; Chapman, K.E. Glucocorticoids, antenatal corticosteroid therapy and fetal heart maturation. J. Mol. Endocrinol. 2018, 61, R61-R73. [CrossRef] 
25. Elwany, E.; Omar, S.; Ahmed, A.; Heba, G.; Atef, D. Antenatal dexamethasone effect on Doppler blood flow velocity in women at risk for preterm birth: Prospective case series. Afr. Health Sci. 2018, 18, 596-600. [CrossRef] [PubMed]

26. Inan, C.; Sayin, N.C.; Dolgun, Z.N.; Erzincan, S.G.; Uzun, I.; Sutcu, H.; Sut, N.; Varol, F.G. Effects of betamethasone on fetoplacental and maternal hemodynamics in preterm pregnancies. Int. J. Gynecol. Obstet. 2018, 141, 354-359. [CrossRef] [PubMed]

27. Mulder, E.J.; de Heus, R.; Visser, G.H. Antenatal corticosteroid therapy: Short-term effects on fetal behaviour and haemodynamics. Semin. Fetal Neonatal Med. 2009, 14, 151-156. [CrossRef]

28. Schaeffer, L.; Burkhardt, T.; Tomaske, M.; Schmidt, S.; Luzi, F.; Rauh, M.; Leone, A.; Beinder, E. Effect of antenatal betamethasone administration on neonatal cardiac autonomic balance. Pediatr. Res. 2010, 68, 286-291. [CrossRef]

29. Bonsante, F.; Latorre, G.; Iacobelli, S.; Forziati, V.; Laforgia, N.; Esposito, L.; Mautone, A. Early Low-Dose Hydrocortisone in Very Preterm Infants: A Randomized, Placebo-Controlled Trial. Neonatology 2006, 91, 217-221. [CrossRef]

30. Bokslag, A.; van Weissenbruch, M.; Mol, B.W.; de Groot, C.J. Preeclampsia; short and long-term consequences for mother and neonate. Early Hum. Dev. 2016, 102, 47-50. [CrossRef]

31. Melamed, N.; Asztalos, E.; Murphy, K.; Zaltz, A.; Redelmeier, D.; Shah, B.R.; Barrett, J. Neurodevelopmental disorders among term infants exposed to antenatal corticosteroids during pregnancy: A population-based study. BMJ Open 2019, 9, e031197. [CrossRef] [PubMed]

32. Räikkönen, K.; Gissler, M.; Kajantie, E. Associations between Maternal Antenatal Corticosteroid Treatment and Mental and Behavioral Disorders in Children. JAMA J. Am. Med. Assoc. 2020, 323, 1924-1933. [CrossRef] [PubMed]

33. Agarwal, P.K.; Xie, H.; Rema, A.S.S.; Rajadurai, V.S.; Lim, S.B.; Meaney, M.; Daniel, L.M. Evaluation of the Ages and Stages Questionnaire (ASQ 3) as a developmental screener at 9, 18, and 24 months. Early Hum. Dev. 2020, 147, 105081. [CrossRef] [PubMed]

34. Fernández, P. Determinación del tamaño muestral. Cad. Aten. Primaria 1996, 3, 1-6. 